\title{
Estimating the Hurst parameter
}

\author{
Corinne Berzin ${ }^{1}$ and José R. León ${ }^{2 *}$
}

${ }^{1}$ LabSad, BSHM, Université Pierre Mendès-France, 1251 Avenue centrale, BP 47, 38040 Grenoble cedex 9, France. email: Corinne.Berzin@upmf-grenoble.fr

${ }^{2}$ Universidad Central de Venezuela, Caracas, Venezuela. email: jleon@euler.ciens.ucv.ve

\begin{abstract}
Let $\left\{b_{H}(t), t \in \mathbb{R}\right\}$ be a fractional Brownian motion with parameter $0<$ $H<1$. We are interested in the estimation of this parameter. To achieve this goal, we consider certain functionals of the second order increments of $b_{H}(\cdot)$, using variation technics. Based on an almost-sure convergence theorem for general functionals, we single out particular functionals that allows to construct certain regression models for the parameter $H$. We show that this regression based estimator for $H$ is asymptotically unbiased, consistent and that it satisfies a Central Limit Theorem (CLT).
\end{abstract}

Key words: central limit theorem, estimation, fractional Brownian motion, Gaussian processes, Hermite polynomials.

\section{Introduction}

In this work, our main interest is to provide estimators of the Hurst parameter $H$ $(0<H<1)$ for a fractional Brownian motion $b_{H}(\cdot)$. Instead of the original process we assume we observe a smoothed by convolution process, defined as $b_{H}^{\varepsilon}(u)=\varphi_{\varepsilon} * b_{H}(u)$. Here $\varepsilon \rightarrow 0$ is the smoothing parameter and $\varphi_{\varepsilon}$ is a positive convolution kernel. Our method consists in obtaining some least squares estimators in certain regression models. The asymptotic behavior of such estimators will be equivalent to those of certain non-linear functionals of the Gaussian process $b_{H}^{\varepsilon}(\cdot)$, whose asymptotic behavior can be obtained by using the method of moments, via the Diagram formula.

The estimation of the Hurst parameter, or of the other parameters associated to long rang dependence, has been recently a matter of intense research. For this different approaches have been developed. Some of them consider spectral methods: as the Whittle estimator [9], [15], and also estimators based on the log-periodogram of the data [12], [14]. Others use variation methods as in [2], [5] and [11], or are based on wavelet techniques [8].

Here the problem is different because we use a mollified version of the original process. Nevertheless, our method can be related with the variation methods. It is

*The research of the second author was suported in part by the project "Modelaje Estocástico Aplicado" of the Agenda Petróleo of FONACIT Venezuela. 
important to mention that the recent works of Coeurjolly [5] and Feuerverger et al. [7], constitute our main sources of inspiration.

Let us introduce the problem. Let $\left\{b_{H}(t), t \in \mathbb{R}\right\}$ be the fractional Brownian motion with parameter $0<H<1$. We study the second order increments of $b_{H}(\cdot)$ and we shall establish in section 3.1.1 an almost-sure convergence in law result stated below in Corollary 3.1:

For all $x \in \mathbb{R}$

$$
\lambda\left\{0 \leqslant u \leqslant 1: \frac{b_{H}(u+2 \varepsilon)-2 b_{H}(u+\varepsilon)+b_{H}(u)}{\varepsilon^{H} v_{2 H} \sqrt{4-2^{2 H}}} \leqslant x\right\} \stackrel{a . s .}{\rightarrow} \operatorname{Pr}\left\{N^{*} \leqslant x\right\},
$$

where $v_{2 H}^{2}=\operatorname{var}\left[b_{H}(1)\right], \lambda$ is the Lebesgue measure and $N^{*}$ is a standard Gaussian random variable.

This result also implies that for smooth functions $f$ with at most polynomial growth, we have :

$$
\int_{0}^{1} f\left(\frac{b_{H}(u+2 \varepsilon)-2 b_{H}(u+\varepsilon)+b_{H}(u)}{\varepsilon^{H} v_{2 H} \sqrt{4-2^{2 H}}}\right) \mathrm{d} u \stackrel{a . s .}{\longrightarrow} \mathrm{E}\left[f\left(N^{*}\right)\right] .
$$

Defining $\varphi=1_{[-1,0]} * 1_{[-1,0]}$ and $b_{H}^{\varepsilon}(u)=\varphi_{\varepsilon} * b_{H}(u)$, where $\varphi_{\varepsilon}(\cdot)=\frac{1}{\varepsilon} \varphi(\dot{\bar{\varepsilon}})$, we have $\varepsilon^{2} \ddot{b}_{H}^{\varepsilon}(u)=b_{H}(u+2 \varepsilon)-2 b_{H}(u+\varepsilon)+b_{H}(u)$. Hence (1) can be written

$$
\int_{0}^{1} f\left(\frac{\varepsilon^{(2-H)} \ddot{b}_{H}^{\varepsilon}(u)}{v_{2 H} \sqrt{4-2^{2 H}}}\right) \mathrm{d} u \stackrel{a . s .}{\longrightarrow} \mathrm{E}\left[f\left(N^{*}\right)\right] .
$$

By considering other regularizations: $b_{H}^{\varepsilon}(u)=\varphi_{\varepsilon} * b_{H}(u), \varphi$ being a $C^{2}$ positive kernel with $L^{1}$ norm equal to one, we can write a result similar to (1) in the form:

$$
\int_{0}^{1} f\left(Z_{\varepsilon}(u)\right) \mathrm{d} u \stackrel{a . s}{\longrightarrow} \mathrm{E}\left[f\left(N^{*}\right)\right]
$$

where $Z_{\varepsilon}(u)=\frac{\varepsilon^{(2-H)} \ddot{b}_{H}^{\varepsilon}(u)}{\sigma_{2 H}}$, with $\sigma_{2 H}^{2}=\operatorname{var}\left[\varepsilon^{(2-H)} \ddot{b}_{H}^{\varepsilon}(u)\right]$.

By observing $b_{H}^{\varepsilon}(u)$ at several scales of the parameter $\varepsilon$, i.e. $h_{i}=\varepsilon c_{i}, c_{i}>0$, $i=1, \ldots, l$, we want to estimate the $H$ parameter. To construct the estimator, we consider $f_{\beta}(x)=|x|^{\beta}$. Then using (2), we get:

$$
\log \left(M_{\beta}(\varepsilon)\right)=(H-2) \beta \log (\varepsilon)+\beta \log \left(\sigma_{2 H}\right)+\log \left(\mathrm{E}\left[\left|N^{*}\right|^{\beta}\right]\right)+o_{a . s .}(1)
$$

where $M_{\beta}(\varepsilon)=\int_{0}^{1}\left|\ddot{b}_{H}^{\varepsilon}(u)\right|^{\beta} \mathrm{d} u$. The following regression model can be written, for each scale $h_{i}$ :

$$
Y_{i}=a X_{i}+b+\xi_{i}, \quad i=1, \ldots, l
$$

where $a=(H-2) \beta$ and for $i=1, \ldots, l, Y_{i}=\log \left(M_{\beta}\left(h_{i}\right)\right), X_{i}=\log \left(h_{i}\right)$ and $b=\beta \log \left(\sigma_{2 H}\right)+\log \left(\mathrm{E}\left[\left|N^{*}\right|^{\beta}\right]\right)$.

Hence, the least squares estimator $\hat{H}_{\beta}$ of $H$ is defined as

$$
\beta\left(\hat{H}_{\beta}-2\right)=\sum_{i=1}^{l} z_{i} \log \left(M_{\beta}\left(h_{i}\right)\right)
$$


where,

$$
z_{i}=\frac{y_{i}}{\sum_{i=1}^{l} y_{i}^{2}}, \quad y_{i}=\log \left(c_{i}\right)-\frac{1}{l} \sum_{i=1}^{l} \log \left(c_{i}\right) .
$$

We shall prove that estimator $\hat{H}_{\beta}$ is strongly consistent, asymptotically unbiased and asymptotically Gaussian. For the later, we need to study the convergence rate in (2) for a function $f \in L^{2}(\phi(x) \mathrm{d} x)$ where $\phi$ stands for the standard Gaussian measure. We can formulate the problem in the following way:

Let $g$ be a function in $L^{2}(\phi(x) \mathrm{d} x)$ and $g(x)=\sum_{n=1}^{\infty} \hat{g}_{n} H_{n}(x)$ its Hermite expansion. We have $\|g\|_{2, \phi}^{2}=\sum_{n=1}^{\infty} \hat{g}_{n}^{2} n !<+\infty$ and $\hat{g}_{0}=0$. We prove in Theorem 3.2 that the functional defined for $c>0$ by

$$
S_{g}(\varepsilon c)=\frac{1}{\sqrt{\varepsilon c}} \int_{0}^{1} g\left(Z_{\varepsilon c}(u)\right) \mathrm{d} u,
$$

converges in distribution towards a cylindrical centered Gaussian process $X(c)$.

The CLT result for $\hat{H}_{\beta}$, follows by considering the asymptotic behavior of the functional associated with $g_{\beta}(x)=|x|^{\beta} / \mathrm{E}\left[\left|N^{*}\right|^{\beta}\right]-1$, and from the fact that $\mid\left(\hat{H}_{\beta}-H\right) / \sqrt{\varepsilon}-$ $\frac{1}{\beta} \sum_{i=1}^{l} z_{i} \sqrt{c_{i}} S_{g_{\beta}}\left(\varepsilon c_{i}\right) \mid=o_{p}(1)$.

This theorem also allows us to consider another least squares estimator of $H$, weakly consistent and unbiased, defined by

$$
\hat{H}_{\log }-2=\sum_{i=1}^{l} z_{i} M_{\log }\left(h_{i}\right), \text { where } M_{\log }(\varepsilon)=\int_{0}^{1} \log \left(\left|\ddot{b}_{H}^{\varepsilon}(u)\right|\right) \mathrm{d} u .
$$

In this case the associated function will be $g_{\log }(x)=\log (|x|)-\mathrm{E}\left[\log \left(\left|N^{*}\right|\right)\right]$.

It is important to mention that working with the second order increments allows us to obtain an asymptotic Gaussian result, for all the possible values of parameter $H$ i.e. $(0,1)$. In previous articles, we considered only the first order increments. This leads to two different results according to whether $H$ belongs to $(0,3 / 4)$ or to $(3 / 4,1)$. In the first case, the limit is Gaussian and in the second one, it belongs to the second Itô-Wiener Chaos. This dichotomy makes the asymptotic behavior more involved.

The paper is organized as follows. In Section 2, we state some notation and the hypothesis under which we work. Section 3 is devoted to establishing the main results. Section 4 contains the proofs.

\section{Hypothesis and notation}

Let $\left\{b_{H}(t), t \in \mathbb{R}\right\}$ be a fractional Brownian motion with parameter $0<H<1$ (see for instance [13]), i.e. $b_{H}(\cdot)$ is a centered Gaussian process with the covariance function:

$$
\mathrm{E}\left[b_{H}(t) b_{H}(s)\right]=\frac{1}{2} v_{2 H}^{2}\left[|t|^{2 H}+|s|^{2 H}-|t-s|^{2 H}\right]
$$

with $v_{2 H}^{2}=[\Gamma(2 H+1) \sin (\pi H)]^{-1}$. We define, for a $C^{2}$ density $\varphi$ with compact support included in $[-1,1]$, for each $t \geqslant 0$ and $\varepsilon>0$ the regularized processes:

$$
b_{H}^{\varepsilon}(t)=\frac{1}{\varepsilon} \int_{-\infty}^{\infty} \varphi\left(\frac{t-x}{\varepsilon}\right) b_{H}(x) \mathrm{d} x \quad \text { and } \quad Z_{\varepsilon}(t)=\frac{\varepsilon^{(2-H)} \ddot{b}_{H}^{\varepsilon}(t)}{\sigma_{2 H}},
$$




$$
\text { with } \sigma_{2 H}^{2}:=\operatorname{var}\left[\varepsilon^{(2-H)} \ddot{b}_{H}^{\varepsilon}(t)\right]=\frac{1}{2 \pi} \int_{-\infty}^{+\infty}|x|^{(3-2 H)}|\hat{\varphi}(-x)|^{2} \mathrm{~d} x .
$$

We can also consider the function $\varphi=1_{[-1,0]} * 1_{[-1,0]}$. Even if it does not belong to the class defined above it is important because the second derivative of the corresponding smoothed process $b_{H}^{\varepsilon}(t)$ gives place to the second order increments for fBm. This assertion is easy to see by using the following representation:

$$
b_{H}^{\varepsilon}(t)=\int_{-2}^{-1}(u+2) b_{H}(t-\varepsilon u) \mathrm{d} u-\int_{-1}^{0} u b_{H}(t-\varepsilon u) \mathrm{d} u .
$$

Moreover, all the results that we shall obtain below could be shown, in a similar form, for this particularly important regularization.

We shall use the Hermite polynomials, which can be defined by $\exp \left(t x-t^{2} / 2\right)=$ $\sum_{n=0}^{\infty} H_{n}(x) t^{n} / n$ !. They form an orthogonal system for the standard Gaussian measure $\phi(x) \mathrm{d} x$ and, if $h \in L^{2}(\phi(x) \mathrm{d} x), h(x)=\sum_{n=0}^{\infty} \hat{h}_{n} H_{n}(x)$ and $\|h\|_{2, \phi}^{2}=\sum_{n=0}^{\infty} \hat{h}_{n}^{2} n !$. Mehler's formula (see [4]) gives a simple form to compute the covariance between two $L^{2}$ functions of Gaussian random variables: if $k \in L^{2}(\phi(x) \mathrm{d} x), k(x)=\sum_{n=0}^{\infty} \hat{k}_{n} H_{n}(x)$ and if $(X, Y)$ is a standard Gaussian random vector with correlation $\rho$ then

$$
\mathrm{E}[h(X) k(Y)]=\sum_{n=0}^{\infty} \hat{h}_{n} \hat{k}_{n} n ! \rho^{n}
$$

Throughout the paper, $\mathbf{C}$ shall stand for a generic constant, whose value may change during a proof, and log for the Naperian logarithm.

$N^{*}$ will denote a standard Gaussian random variable, and the symbol $\Rightarrow$ will mean weak convergence of measures.

\section{Results}

In this section we shall establish the main results of this work. For this we need to introduce some definitions.

Let $g$ be a function in $L^{2}(\phi(x) \mathrm{d} x)$ such that $g(x)=\sum_{n=1}^{\infty} \hat{g}_{n} H_{n}(x)$, with $\|g\|_{2, \phi}^{2}=$ $\sum_{n=1}^{\infty} \hat{g}_{n}^{2} n !<+\infty$

We define, for $x \in \mathbb{R}$,

$$
\begin{aligned}
\rho_{H}(x) & =\mathrm{E}\left[Z_{\varepsilon}(\varepsilon x+u) Z_{\varepsilon}(u)\right]=\frac{-v_{2 H}^{2}}{2 \sigma_{2 H}^{2}} \int_{-\infty}^{\infty} \ddot{\varphi} * \tilde{\varphi}(y)|x-y|^{2 H} \mathrm{~d} y \\
& =\frac{1}{2 \pi \sigma_{2 H}^{2}} \int_{-\infty}^{\infty}|y|^{(3-2 H)} e^{i x y}|\widehat{\varphi}(-y)|^{2} \mathrm{~d} y
\end{aligned}
$$

and $\widetilde{\varphi}(y)=\ddot{\varphi}(-y)$. The fact that $\rho_{H}$ does not depend on the variable $u$ follows from $\varphi \in C^{2}$ and the stationary increments of $b_{H}$.

We shall write

$$
\sigma_{g}^{2}=\sum_{n=1}^{\infty} \hat{g}_{n}^{2} n ! \int_{-\infty}^{+\infty} \rho_{H}^{n}(x) \mathrm{d} x
$$


More generally, for $x \in \mathbb{R}$ and $b, c>0$, we define

$$
\begin{aligned}
\rho_{H}(x, b, c) & =\mathrm{E}\left[Z_{\varepsilon b}(\varepsilon x+u) Z_{\varepsilon c}(u)\right] \\
& =\left(\frac{-v_{2 H}^{2}}{2 \sigma_{2 H}^{2}}\right)\left(\frac{b}{c}\right)^{(H+1)} \int_{-\infty}^{\infty} \int_{-\infty}^{\infty} \ddot{\varphi}(z) \ddot{\varphi}\left(\frac{b}{c}(z-y)\right)\left|\frac{x}{b}-y\right|^{2 H} \mathrm{~d} z \mathrm{~d} y \\
& =\frac{(b c)^{(2-H)}}{2 \pi \sigma_{2 H}^{2}} \int_{-\infty}^{\infty}|y|^{(3-2 H)} e^{i x y} \widehat{\varphi}(-b y) \widehat{\varphi}(c y) \mathrm{d} y=\rho_{H}(-x, c, b),
\end{aligned}
$$

and

$$
\rho_{g}(b, c)=\frac{1}{\sqrt{b c}} \sum_{n=1}^{\infty} \hat{g}_{n}^{2} n ! \int_{-\infty}^{+\infty} \rho_{H}^{n}(x, b, c) \mathrm{d} x .
$$

Note that $\rho_{H}(x, c, c)=\rho_{H}\left(\frac{x}{c}\right)$ and then $\rho_{g}(c, c)=\sigma_{g}^{2}$.

For all $m \in \mathbb{N}^{*}$, for all $c_{1}>0, c_{2}>0, \ldots, c_{m}>0$ and for all $d_{1}, d_{2}, \ldots, d_{m} \in \mathbb{R}$, we will denote

$$
\sigma_{g, m}^{2}(\mathbf{c}, \mathbf{d})=\sum_{i=1}^{m} \sum_{j=1}^{m} d_{i} d_{j} \rho_{g}\left(c_{i}, c_{j}\right) .
$$

For $\varepsilon>0$, define

$$
S_{g}(\varepsilon)=\varepsilon^{-\frac{1}{2}} \int_{0}^{1} g\left(Z_{\varepsilon}(u)\right) \mathrm{d} u .
$$

\subsection{Convergence for $\left(Z_{\varepsilon}(u)\right)_{u \in[0,1]}$ and $\left(S_{g}(\varepsilon c)\right)_{c>0}$}

\subsubsection{Almost sure convergence}

Theorem 3.1 For all $0<H<1$ and $k \in \mathbb{N}^{*}$,

$$
\int_{0}^{1}\left[Z_{\varepsilon}(u)\right]^{k} \mathrm{~d} u \underset{\varepsilon \rightarrow 0}{\stackrel{a . s .}{\rightarrow}} \mathrm{E}\left[N^{*}\right]^{k} .
$$

Corollary 3.1 Almost surely, for all $0<H<1$,

$$
Z_{\varepsilon}(\cdot) \underset{\varepsilon \rightarrow 0}{\Rightarrow} N^{*}
$$

The above convergence is in law, the random variable $Z_{\varepsilon}(\cdot)$ is considered as a variable on $([0,1], \lambda)$ where $\lambda$ is the Lebesgue measure.

Remark : For the first order increments, a similar theorem was proved by Azaïs \& Wschebor in [1].

\subsubsection{Convergence in law}

Theorem 3.2 For all $0<H<1$,

$$
S_{g}(\varepsilon \cdot) \underset{\varepsilon \rightarrow 0}{\Rightarrow} X(\cdot),
$$

where $X(\cdot)$ is a cylindrical centered Gaussian process with covariance $\rho_{g}(b, c)=$ $\mathrm{E}[X(b) X(c)], b, c>0$.

The above convergence is in the sense of finite-dimensional distributions. 
Remark 1 : If $c>0$ is fixed, $S_{g}(\varepsilon c) \underset{\varepsilon \rightarrow 0}{\Rightarrow} \sigma_{g} N^{*}$. This particular result was shown in Corollary 3.2 (i) in C. Berzin and J.R. León [3].

In section 4.2.1 we shall prove the following lemma

Lemma 3.1 For all $m \in \mathbb{N}^{*}$, for all $c_{1}>0, \cdots, c_{m}>0$ and $d_{1}, \cdots, d_{m} \in \mathbb{R}$, $\sigma_{g, m}^{2}(\mathbf{c}, \mathbf{d})=\lim _{\varepsilon \rightarrow 0} \mathrm{E}\left[\sum_{i=1}^{m} d_{i} S_{g}\left(\varepsilon c_{i}\right)\right]^{2} \geqslant 0$.

Remark 2: The above lemma allows us to conclude that $\rho_{g}(b, c)$ is a covariance function, since it is a symmetrical function in $b$ and $c$.

\subsubsection{Estimation of the Hurst parameter}

Let $\beta>0$ and

$$
M_{\beta}(\varepsilon)=\int_{0}^{1}\left|\ddot{b}_{H}^{\varepsilon}(u)\right|^{\beta} \mathrm{d} u .
$$

Thanks to Theorem 3.1 and Corollary 3.1,

$$
\int_{0}^{1}\left|Z_{\varepsilon}(u)\right|^{\beta} \mathrm{d} u=\frac{\varepsilon^{(2-H) \beta}}{\sigma_{2 H}^{\beta}} M_{\beta}(\varepsilon) \underset{\varepsilon \rightarrow 0}{\stackrel{a . s .}{\rightarrow}} \mathrm{E}\left[\left|N^{*}\right|^{\beta}\right] .
$$

Then,

$$
(2-H) \beta \log (\varepsilon)-\beta \log \left(\sigma_{2 H}\right)+\log \left(M_{\beta}(\varepsilon)\right) \underset{\varepsilon \rightarrow 0}{\stackrel{a . s .}{\rightarrow}} \log \left(\mathrm{E}\left[\left|N^{*}\right|^{\beta}\right]\right) .
$$

Thus

$$
\begin{aligned}
\log \left(M_{\beta}(\varepsilon)\right)= & (H-2) \beta \log (\varepsilon) \\
& +\beta \log \left(\sigma_{2 H}\right)+\log \left(\mathrm{E}\left[\left|N^{*}\right|^{\beta}\right]\right)+o_{\text {a.s. }}(1) .
\end{aligned}
$$

Let $h_{i}=\varepsilon c_{i}, c_{i}>0, i=1, \ldots, l$. The model can be written as:

$$
Y_{i}=a X_{i}+b+\xi_{i}, \quad i=1, \ldots, l
$$

where $a=(H-2) \beta$ and for $i=1, \ldots, l, Y_{i}=\log \left(M_{\beta}\left(h_{i}\right)\right), X_{i}=\log \left(h_{i}\right)$ and $b=\beta \log \left(\sigma_{2 H}\right)+\log \left(\mathrm{E}\left[\left|N^{*}\right|^{\beta}\right]\right)$.

Hence, the least squares estimator $\hat{H}_{\beta}$ of $H$ is given by

$$
\beta\left(\hat{H}_{\beta}-2\right)=\sum_{i=1}^{l} z_{i} \log \left(M_{\beta}\left(h_{i}\right)\right),
$$

where for $i=1, \ldots, l, z_{i}=\frac{y_{i}}{\sum_{i=1}^{l} y_{i}^{2}}$ and $y_{i}=\log \left(c_{i}\right)-\frac{1}{l} \sum_{i=1}^{l} \log \left(c_{i}\right)$.

Note the following property

$$
\sum_{i=1}^{l} y_{i}=0 \text { and } \sum_{i=1}^{l} z_{i} y_{i}=1 .
$$

We have the following corollary of Theorem 3.2. 
Corollary $3.2 \quad$ 1) $\hat{H}_{\beta}$ is an asymptotically unbiased strongly consistent estimator of $H$.

2) Furthermore,

$$
\frac{1}{\sqrt{\varepsilon}}\left(\hat{H}_{\beta}-H\right) \underset{\varepsilon \rightarrow 0}{\Rightarrow} \mathcal{N}\left(0, \sigma_{g_{\beta}, l}^{2}(\mathbf{c}, \sqrt{\mathbf{c}}(\mathbf{z} / \beta))\right.
$$

where

$$
g_{\beta}(x)=\frac{|x|^{\beta}}{\mathrm{E}\left[\left|N^{*}\right|^{\beta}\right]}-1=\sum_{n=1}^{\infty} \hat{g}_{2 n, \beta} H_{2 n}(x),
$$

with

$$
\begin{aligned}
\hat{g}_{2 n, \beta} & =\sum_{p=0}^{n} \frac{(-1)^{(n-p)}}{(2 p) !(n-p) ! 2^{(n-2 p)}} \frac{\Gamma\left(p+\left(\frac{\beta+1}{2}\right)\right)}{\Gamma\left(\frac{\beta+1}{2}\right)} \\
& =\frac{1}{(2 n) !} \prod_{i=0}^{n-1}(\beta-2 i) .
\end{aligned}
$$

Remark 1: As in [5], the variance $\sigma_{g_{\beta}, l}^{2}(\mathbf{c}, \sqrt{\mathbf{c}}(\mathbf{z} / \beta))$ is minimal for $\beta=2$. This fact will be shown in section 4.3.1 after the proof of Corollary 3.2.

Remark 2: Note that for $\beta=1, M_{1}(\varepsilon)=\int_{0}^{1}\left|\ddot{b}_{H}^{\varepsilon}(u)\right| \mathrm{d} u=\int_{-\infty}^{+\infty} N_{[0,1]}^{\dot{b}_{H}^{\varepsilon}}(x) \mathrm{d} x$, where $N_{[0,1]}^{\dot{b}_{H}^{\varepsilon}}(x)$ denotes the number of times the process $\dot{b}_{H}^{\varepsilon}(\cdot)$ crosses level $x$ before time 1. This remark allows to establish a link between our estimator and that defined in [7], but in this work the authors use the crossings of $b_{H}^{\varepsilon}(\cdot)$ instead of those of $\dot{b}_{H}^{\varepsilon}(\cdot)$.

Now, let us define

$$
M_{\log }(\varepsilon)=\int_{0}^{1} \log \left(\left|\ddot{b}_{H}^{\varepsilon}(u)\right|\right) \mathrm{d} u .
$$

Lemma 3.1 following Theorem 3.2, also entails that

$$
\int_{0}^{1} \log \left(\left|Z_{\varepsilon}(u)\right|\right) \mathrm{d} u \underset{\varepsilon \rightarrow 0}{\stackrel{\mathcal{P}}{\longrightarrow}} \mathrm{E}\left[\log \left(\left|N^{*}\right|\right)\right],
$$

and also in $L^{2}(\Omega)$.

We proceed as before and the least squares estimator $\hat{H}_{\log }$ of $H$ is given by

$$
\hat{H}_{\log }-2=\sum_{i=1}^{l} z_{i} M_{\log }\left(h_{i}\right) .
$$

Theorem 3.2 gives us the following corollary.

Corollary $3.3 \quad$ 1) $\hat{H}_{\log }$ is an unbiased weakly consistent estimator of $H$.

2) Furthermore,

$$
\frac{1}{\sqrt{\varepsilon}}\left(\hat{H}_{\log }-H\right) \underset{\varepsilon \rightarrow 0}{\Rightarrow} \mathcal{N}\left(0, \sigma_{g_{\log }, l}^{2}(\mathbf{c}, \sqrt{\mathbf{c}} \mathbf{z})\right)
$$


where

$$
g_{\log }(x)=\log (|x|)-\mathrm{E}\left[\log \left(\left|N^{*}\right|\right)\right]=\sum_{n=1}^{\infty} \hat{g}_{2 n, \log } H_{2 n}(x),
$$

with

$$
\hat{g}_{2 n, \log }=\frac{(-1)^{(n-1)}}{2 n(2 n) ! !} .
$$

We can link the two estimators $\hat{H}_{\beta}$ and $\hat{H}_{\log }$. For this, let $\beta(\varepsilon)$ a sequence of positive numbers going to zero when $\varepsilon$ goes to zero and let $\hat{H}_{\beta(\varepsilon)}$ be the corresponding estimator say

$$
\begin{aligned}
\hat{H}_{\beta(\varepsilon)}-2 & =\sum_{i=1}^{l} z_{i} \frac{\log \left(M_{\beta\left(h_{i}\right)}\left(h_{i}\right)\right)}{\beta\left(h_{i}\right)} \\
\text { where } M_{\beta(\varepsilon)}(\varepsilon) & =\int_{0}^{1}\left|\ddot{b}_{H}^{\varepsilon}(u)\right|^{\beta(\varepsilon)} \mathrm{d} u .
\end{aligned}
$$

We have the following corollary.

Corollary 3.4 If $\beta(\varepsilon)=o(\sqrt{\varepsilon})$ then $\hat{H}_{\beta(\varepsilon)}$ is an asymptotically unbiased weakly consistent estimator of $H$ and the asymptotic behavior of $\frac{1}{\sqrt{\varepsilon}}\left(\hat{H}_{\beta(\varepsilon)}-H\right)$ is the same one as that of $\frac{1}{\sqrt{\varepsilon}}\left(\hat{H}_{\log }-H\right)$.

\section{Proofs}

\subsection{Almost sure convergence for $\left(Z_{\varepsilon}(u)\right)_{u \in[0,1]}$}

Proof of Theorem 3.1. For all $k \in \mathbb{N}^{*}$,

$$
\mathrm{E}\left[\int_{0}^{1}\left[Z_{\varepsilon}(u)\right]^{k} \mathrm{~d} u\right]=\mathrm{E}\left[N^{*}\right]^{k}
$$

Furthermore,

$$
\operatorname{var}\left[\int_{0}^{1}\left[Z_{\varepsilon}(u)\right]^{k} \mathrm{~d} u\right]=\mathrm{E}\left[\int_{0}^{1}\left(\left[Z_{\varepsilon}(u)\right]^{k}-\mathrm{E}\left[N^{*}\right]^{k}\right) \mathrm{d} u\right]^{2} .
$$

Let $g_{(k)}(x)=x^{k}-\mathrm{E}\left[N^{*}\right]^{k}=\sum_{n=1}^{\infty} \hat{g}_{n,(k)} H_{n}(x)$. Mehler's formula (3) entails that

$$
\begin{aligned}
\operatorname{var}\left[\int_{0}^{1}\left[Z_{\varepsilon}(u)\right]^{k} \mathrm{~d} u\right] & =\sum_{n=1}^{\infty} \hat{g}_{n,(k)}^{2} n ! \int_{0}^{1} \int_{0}^{1} \rho_{H}^{n}\left(\frac{u-v}{\varepsilon}\right) \mathrm{d} u \mathrm{~d} v \\
& =\sum_{n=1}^{\infty} \hat{g}_{n,(k)}^{2} n ! \int_{-1}^{1}(1-|u|) \rho_{H}^{n}\left(\frac{u}{\varepsilon}\right) \mathrm{d} u .
\end{aligned}
$$


If we let $u=\varepsilon x$, we get

$$
\operatorname{var}\left[\int_{0}^{1}\left[Z_{\varepsilon}(u)\right]^{k} \mathrm{~d} u\right]=\varepsilon \sum_{n=1}^{\infty} \hat{g}_{n,(k)}^{2} n ! \int_{-\frac{1}{\varepsilon}}^{\frac{1}{\varepsilon}}(1-\varepsilon|x|) \rho_{H}^{n}(x) \mathrm{d} x .
$$

Since $\left|\rho_{H}(x)\right| \leqslant 1,\left|\rho_{H}^{n}(x)\right| \leqslant\left|\rho_{H}(x)\right|$ for $n \geqslant 1$, we have

$$
\begin{aligned}
\operatorname{var}\left[\int_{0}^{1}\left[Z_{\varepsilon}(u)\right]^{k} \mathrm{~d} u\right] & \leqslant \varepsilon\left(\sum_{n=1}^{\infty} \hat{g}_{n,(k)}^{2} n !\right) \int_{-\frac{1}{\varepsilon}}^{\frac{1}{\varepsilon}}\left|\rho_{H}(x)\right| \mathrm{d} x \\
& \leqslant \varepsilon \|\left. g_{(k)}\right|_{2, \phi} ^{2} \int_{-\frac{1}{\varepsilon}}^{\frac{1}{\varepsilon}}\left|\rho_{H}(x)\right| \mathrm{d} x
\end{aligned}
$$

A Taylor expansion of order four of the function $(1-x)^{2 H}$ and integration by parts show that $\rho_{H}(x)$ is equivalent to $-v_{2 H}^{2} / \sigma_{2 H}^{2}|x|^{(2 H-4)} 2 H(2 H-1)(H-1)(2 H-3)$ for large values of $|x|$. Thus $\left|\rho_{H}(x)\right|$ is bounded from above by $\mathbf{C}|x|^{(2 H-4)}$. As $\left\|g_{(k)}\right\|_{2, \phi}^{2}<+\infty$, we get

$$
\operatorname{var}\left[\int_{0}^{1}\left[Z_{\varepsilon}(u)\right]^{k} \mathrm{~d} u\right] \leqslant \mathbf{C} \varepsilon .
$$

Now let $\varepsilon_{\nu}=\nu^{-a}, a>1$. Using the Borel-Cantelli Lemma, one has

$$
\int_{0}^{1}\left[Z_{\varepsilon_{\nu}}(u)\right]^{k} \mathrm{~d} u \underset{\nu \rightarrow \infty}{\stackrel{a . s .}{\rightarrow}} \mathrm{E}\left[N^{*}\right]^{k} .
$$

For $\varepsilon_{\nu+1} \leqslant \varepsilon \leqslant \varepsilon_{\nu}$ let us consider the modulus of the difference

$$
\left|\int_{0}^{1}\left[Z_{\varepsilon_{\nu}}(u)\right]^{k} \mathrm{~d} u-\int_{0}^{1}\left[Z_{\varepsilon}(u)\right]^{k} \mathrm{~d} u\right| .
$$

One has

$$
\left|\int_{0}^{1}\left[Z_{\varepsilon_{\nu}}(u)\right]^{k} \mathrm{~d} u-\int_{0}^{1}\left[Z_{\varepsilon}(u)\right]^{k} \mathrm{~d} u\right| \leqslant J_{1}+J_{2},
$$

where

$$
J_{1}=\left[1-\left(\frac{\varepsilon}{\varepsilon_{\nu}}\right)^{(2-H) k}\right]\left|\int_{0}^{1}\left[Z_{\varepsilon_{\nu}}(u)\right]^{k} \mathrm{~d} u\right|
$$

and

$$
J_{2}=\left|\left(\frac{\varepsilon}{\varepsilon_{\nu}}\right)^{(2-H) k} \int_{0}^{1}\left[Z_{\varepsilon_{\nu}}(u)\right]^{k} \mathrm{~d} u-\int_{0}^{1}\left[Z_{\varepsilon}(u)\right]^{k} \mathrm{~d} u\right| .
$$


We study $J_{1}$ and $J_{2}$ separately. Using (15), one has

$$
\sup _{\varepsilon_{\nu+1} \leqslant \varepsilon \leqslant \varepsilon_{\nu}} J_{1} \leqslant\left[1-\left(\frac{\varepsilon_{\nu+1}}{\varepsilon_{\nu}}\right)^{(2-H) k}\right]\left|\int_{0}^{1}\left[Z_{\varepsilon_{\nu}}(u)\right]^{k} \mathrm{~d} u\right| \underset{\nu \rightarrow \infty}{\stackrel{a . s .}{\rightarrow}} 0 .
$$

Moreover, for $J_{2}$ we have

$$
J_{2} \leqslant\left(\frac{1}{\sigma_{2 H}}\right)^{k} \varepsilon^{(2-H) k} \int_{0}^{1}\left|\left[\ddot{b}_{H}^{\varepsilon_{\nu}}(u)\right]^{k}-\left[\ddot{b}_{H}^{\varepsilon}(u)\right]^{k}\right| \mathrm{d} u .
$$

Now

$$
\left[\ddot{b}_{H}^{\varepsilon}(u)\right]^{k}-\left[\ddot{b}_{H}^{\varepsilon_{\nu}}(u)\right]^{k}=\left(\ddot{b}_{H}^{\varepsilon}(u)-\ddot{b}_{H}^{\varepsilon_{\nu}}(u)\right)\left(\sum_{j=0}^{k-1}\left[\ddot{b}_{H}^{\varepsilon}(u)\right]^{j}\left[\ddot{b}_{H}^{\varepsilon_{\nu}}(u)\right]^{(k-1-j)}\right) .
$$

Using that the trajectories of $b_{H}(\cdot)$ are $(H-\delta)$-Hölder continuous, for any $\delta>0$ i.e.

$$
\left|b_{H}(u+\varepsilon)-b_{H}(u)\right| \leqslant \mathbf{C}(\omega) \varepsilon^{(H-\delta)},
$$

we get

$$
\left|\ddot{b}_{H}^{\varepsilon}(u)\right|=\left|\frac{1}{\varepsilon^{2}} \int_{-\infty}^{\infty} \ddot{\varphi}(v)\left(b_{H}(u-\varepsilon v)-b_{H}(u)\right) d v\right| \leqslant \mathbf{C} \varepsilon^{(H-2-\delta)},
$$

and by similar computations

$$
\left|\ddot{b}_{H}^{\varepsilon}(u)-\ddot{b}_{H}^{\varepsilon_{\nu}}(u)\right| \leqslant \mathbf{C} \varepsilon_{\nu}^{(H-\delta)} \varepsilon^{-2}\left|1-\frac{\varepsilon_{\nu+1}}{\varepsilon_{\nu}}\right|^{(H-\delta)} .
$$

Thus

$$
\left|\left[\ddot{b}_{H}^{\varepsilon}(u)\right]^{k}-\left[\ddot{b}_{H}^{\varepsilon_{\nu}}(u)\right]^{k}\right| \leqslant \mathbf{C} \varepsilon_{\nu}^{(H-\delta)} \varepsilon^{-2}\left|1-\frac{\varepsilon_{\nu+1}}{\varepsilon_{\nu}}\right|^{(H-\delta)} \varepsilon^{(H-2-\delta)(k-1)},
$$

and then

$$
\sup _{\varepsilon_{\nu+1} \leqslant \varepsilon \leqslant \varepsilon_{\nu}} J_{2} \leqslant \mathbf{C}\left(\frac{1}{\sigma_{2 H}}\right)^{k} \varepsilon_{\nu+1}^{-\delta k}\left|1-\frac{\varepsilon_{\nu}}{\varepsilon_{\nu+1}}\right|^{(H-\delta)} \underset{\nu \rightarrow \infty}{\stackrel{a . s .}{\rightarrow}} 0
$$

as soon as $\delta<\frac{H}{(a k+1)}$.

Thus we have proved that

$$
\sup _{\varepsilon_{\nu+1} \leqslant \varepsilon \leqslant \varepsilon_{\nu}}\left|\int_{0}^{1}\left[Z_{\varepsilon_{\nu}}(u)\right]^{k} \mathrm{~d} u-\int_{0}^{1}\left[Z_{\varepsilon}(u)\right]^{k} \mathrm{~d} u\right|
$$

tends almost surely to zero when $\nu$ goes to infinity and Theorem 3.1 follows. Corollary 3.1 follows readily from Theorem 3.1 by the method of moments. 


\subsection{Convergence in law for $\left(S_{g}(\varepsilon c)\right)_{c>0}$}

\subsubsection{Asymptotic variance of $\sum_{i=1}^{m} d_{i} S_{g}\left(\varepsilon c_{i}\right)$}

Proof of Lemma 3.1. Fix $m \in \mathbb{N}^{*}, c_{1}>0, \ldots, c_{m}>0$ and $d_{1}, \ldots, d_{m} \in \mathbb{R}$

$$
\mathrm{E}\left[\sum_{i=1}^{m} d_{i} S_{g}\left(\varepsilon c_{i}\right)\right]^{2}=\sum_{i=1}^{m} \sum_{j=1}^{m} d_{i} d_{j} \mathrm{E}\left[S_{g}\left(\varepsilon c_{i}\right) S_{g}\left(\varepsilon c_{j}\right)\right] .
$$

For fixed $b>0$ and $c>0$ and by Mehler's formula (3), we get as in Section 4.1

$$
\begin{aligned}
\mathrm{E}\left[S_{g}(\varepsilon b) S_{g}(\varepsilon c)\right] & =\frac{1}{\varepsilon \sqrt{b c}} \sum_{n=1}^{\infty} \hat{g}_{n}^{2} n ! \int_{0}^{1} \int_{0}^{1}\left[\rho_{H}\left(\frac{u-v}{\varepsilon}, b, c\right)\right]^{n} \mathrm{~d} u \mathrm{~d} v \\
& =\frac{1}{\sqrt{b c}} \sum_{n=1}^{\infty} \hat{g}_{n}^{2} n ! \int_{-\frac{1}{\varepsilon}}^{\frac{1}{\varepsilon}}(1-\varepsilon|x|)\left[\rho_{H}(x, b, c)\right]^{n} \mathrm{~d} x .
\end{aligned}
$$

Since $\rho_{H}(x, b, c)$ is a correlation we have $\left|\rho_{H}(x, b, c)\right|^{n} \leqslant\left|\rho_{H}(x, b, c)\right|$. Moreover, as for $\rho_{H}(x)$ in section 4.1 it can be seen that $\rho_{H}(x, b, c)$ is equivalent to $-v_{2 H}^{2} / \sigma_{2 H}^{2}|x|^{(2 H-4)}(b c)^{(2-H)} 2 H(2 H-$ $1)(H-1)(2 H-3)$ for $|x|$ large enough. Thus $\left|\rho_{H}(x, b, c)\right|$ is bounded from above by C $|x|^{(2 H-4)}$, for $|x|$ large enough. The dominated convergence theorem entails that

$$
\mathrm{E}\left[S_{g}(\varepsilon b) S_{g}(\varepsilon c)\right] \underset{\varepsilon \rightarrow 0}{\longrightarrow} \rho_{g}(b, c) \text { thus } \mathrm{E}\left[\sum_{i=1}^{m} d_{i} S_{g}\left(\varepsilon c_{i}\right)\right]^{2} \underset{\varepsilon \rightarrow 0}{\longrightarrow} \sigma_{g, m}^{2}(\mathbf{c}, \mathbf{d})
$$

this yields Remark 2 of Theorem 3.2.

\subsubsection{Convergence in law}

Proof of Theorem 3.2. Fix $m \in \mathbb{N}^{*}, c_{1}>0, c_{2}>0, \ldots, c_{m}>0$ and $d_{1}, d_{2}, \ldots$, $d_{m} \in \mathbb{R}$ and

$$
S_{g}(\varepsilon \mathbf{c}):=\sum_{i=1}^{m} d_{i} S_{g}\left(\varepsilon c_{i}\right)
$$

We want to prove that

$$
S_{g}(\varepsilon \mathbf{c}) \underset{\varepsilon \rightarrow 0}{\Rightarrow} \mathcal{N}\left(0 ; \sigma_{g, m}^{2}(\mathbf{c}, \mathbf{d})\right)
$$

Let

$$
S_{g_{M}}(\varepsilon \mathbf{c})=\sum_{i=1}^{m} d_{i} S_{g_{M}}\left(\varepsilon c_{i}\right) \text { with } g_{M}(x)=\sum_{n=1}^{M} \hat{g}_{n} H_{n}(x) .
$$

First, let us prove the following lemma.

\section{Lemma 4.1}

$$
S_{g_{M}}(\varepsilon \mathbf{c}) \underset{\varepsilon \rightarrow 0}{\Rightarrow} \mathcal{N}\left(0 ; \sigma_{g_{M}, m}^{2}(\mathbf{c}, \mathbf{d})\right)
$$


Proof of Lemma 4.1. Let fixed $p \in \mathbb{N}^{*}$, we want to study the asymptotic behavior of $\mathrm{E}\left[S_{g_{M}}(\varepsilon \mathbf{c})\right]^{p}$. For $p=1$, by Mehler's formula (see $\left.(3)\right), \mathrm{E}\left[S_{g_{M}}(\varepsilon \mathbf{c})\right]=0$. Now, for $p \in \mathbb{N}^{*}-\{1\}$,

$$
\begin{aligned}
& \mathrm{E}\left[S_{g_{M}}(\varepsilon \mathbf{c})\right]^{p}=\frac{1}{\varepsilon^{p / 2}} \sum_{j_{1}, \ldots, j_{p}=1}^{m} \frac{d_{j_{1}}}{\sqrt{c_{j_{1}}}} \cdots \frac{d_{j_{p}}}{\sqrt{c_{j_{p}}}} \sum_{l_{1}, \ldots, l_{p}=1}^{M} \hat{g}_{l_{1}} \cdots \hat{g}_{l_{p}} \\
& \times \int_{0}^{1} \int_{0}^{1} \cdots \int_{0}^{1} \mathrm{E}\left[H_{l_{1}}\left(Z_{\varepsilon c_{j_{1}}}\left(s_{1}\right)\right) \cdots H_{l_{p}}\left(Z_{\varepsilon c_{j_{p}}}\left(s_{p}\right)\right)\right] \mathrm{d} \mathbf{s},
\end{aligned}
$$

where $\mathrm{d} \mathbf{s}=\mathrm{d} s_{1} \ldots \mathrm{d} s_{p}$.

To get the asymptotic behavior of the later expression we shall use the diagram formula. Therefore, we must introduce some definitions given in [4]. Besides that our problem is in continuous time and non-ergodic (the time scale does not tend to infinity), we can say that our theorem is a variant of the main result in that work. Nevertheless, we have decided to include the proof for completeness and to make the reading more easy .

An undirected graph $G$ with $l_{1}+l_{2}+\ldots+l_{p}$ vertices is a diagram of order $\left(l_{1}, \ldots, l_{p}\right)$ if:

(i) The set of vertices $V$ of the graph $G$ has the form: $V=\bigcup_{j=1}^{p} L_{j}$ where $L_{j}=\left\{(j, l): 1 \leqslant l \leqslant l_{j}\right\}$ for $j=1, \ldots, p$.

(ii) Each vertex is of degree 1.

(iii) Edges may pass only between different levels.

We denote $\Gamma=\Gamma\left(l_{1}, \ldots, l_{p}\right)$ the set of diagrams having these properties, $G(V)$ denotes the set of edges of $G$; the edges $w$ are oriented, beginning in $d_{1}(w)$ and finishing in $d_{2}(w)$.

The diagram formula [4] allows to write:

$$
\begin{aligned}
& \mathrm{E}\left[H_{l_{1}}\left(Z_{\varepsilon c_{j_{1}}}\left(s_{1}\right)\right) \cdots H_{l_{p}}\left(Z_{\varepsilon c_{j_{p}}}\left(s_{p}\right)\right)\right] \\
&=\sum_{G \in \Gamma} \prod_{w \in G(V)} \prod_{d_{1}(w)<d_{2}(w)} \rho_{H}\left(\frac{s_{d_{1}(w)}-s_{d_{2}(w)}}{\varepsilon}, c_{j_{d_{1}(w)}}, c_{j_{d_{2}(w)}}\right)
\end{aligned}
$$

where $G$ is an undirected graph with $l_{1}+\cdots+l_{p}$ vertices and $p$ levels.

A diagram is said "regular" ([4], p.432) if its levels can be matched in such a way that no edge passes between levels in different pairs, otherwise, it is said "irregular".

We shall classify the diagrams of $\Gamma$ as in [10], p. 1166, calling $R$ the set of the regular graphs and $R^{c}$ the rest.

We start by considering $R$.

Consider a regular diagram $G^{*}$ and let $i$ be the permutation such that

$$
(i(1), i(2)), \ldots,(i(p-1), i(p))
$$

defines the diagram with $p=2 q$. The contribution of this diagram is

$$
\begin{aligned}
I_{m, p}(\varepsilon)\left(G^{*}\right):= & \sum_{j_{1}, \ldots, j_{p}=1}^{m}\left(\prod_{i=1}^{p} \frac{d_{j_{i}}}{\sqrt{c_{j_{i}}}}\right) \\
& \times \prod_{k=1}^{q}\left[\hat{g}_{e(k)}^{2} \frac{1}{\varepsilon} \int_{[0,1]^{2}}\left[\rho_{H}\left(\frac{u-v}{\varepsilon}, c_{j_{i(2 k-1)}}, c_{j_{i(2 k)}}\right)\right]^{e(k)} \mathrm{d} u \mathrm{~d} v\right],
\end{aligned}
$$


where $e(k)$ is the number of edges linking $i(2 k-1)$ to $i(2 k)$.

As in Section 4.2, we get

$$
\begin{aligned}
I_{m, p}(\varepsilon)\left(G^{*}\right)= & \sum_{j_{1}, \ldots, j_{p}=1}^{m}\left(\prod_{i=1}^{p} \frac{d_{j_{i}}}{\sqrt{c_{j_{i}}}}\right) \\
& \times \prod_{k=1}^{q}\left[\hat{g}_{e(k)}^{2} \int_{-\frac{1}{\varepsilon}}^{\frac{1}{\varepsilon}}(1-\varepsilon|x|)\left[\rho_{H}\left(x, c_{j_{i(2 k-1)}}, c_{j_{i(2 k)}}\right)\right]^{e(k)} \mathrm{d} x\right] .
\end{aligned}
$$

Since $\rho_{H}(x, b, c)$ is bounded from above by $\mathbf{C}|x|^{2 H-4}$, for large enough $|x|,\left\|g_{M}\right\|_{2, \phi}^{2}<$ $+\infty$ and $\left|\rho_{H}(x, b, c)\right| \leqslant 1$

$$
\begin{aligned}
I_{m, p}(\varepsilon)\left(G^{*}\right) \underset{\varepsilon \rightarrow 0}{\longrightarrow} & I_{m, p}\left(G^{*}\right):=\sum_{j_{1}, \ldots, j_{p}=1}^{m}\left(\prod_{i=1}^{p} \frac{d_{j_{i}}}{\sqrt{c_{j_{i}}}}\right) \\
& \times \prod_{k=1}^{q}\left[\hat{g}_{e(k)}^{2} \int_{-\infty}^{\infty}\left[\rho_{H}\left(x, c_{j_{i(2 k-1)}}, c_{\left.j_{i(2 k)}\right)}\right)\right]^{e(k)} \mathrm{d} x\right] .
\end{aligned}
$$

The right hand term can be written

$I_{m, p}\left(G^{*}\right)=$

$$
\prod_{k=1}^{q}\left[\hat{g}_{e(k)}^{2} \sum_{j_{i(2 k)}=1}^{m} \sum_{j_{i(2 k-1)=1}}^{m} \frac{d_{j_{i(2 k)}}}{\sqrt{c_{j_{i(2 k)}}}} \frac{d_{j_{i(2 k-1)}}}{\sqrt{c_{j_{i(2 k-1)}}}} \int_{-\infty}^{\infty}\left[\rho_{H}\left(x, c_{j_{i(2 k-1)}}, c_{j_{i(2 k)}}\right)\right]^{e(k)} \mathrm{d} x\right] .
$$

Since we show in Lemma 4.2 that the contribution of the irregular diagrams tends to zero, we obtain that

$$
\lim _{\varepsilon \rightarrow 0}\left[S_{g M}(\varepsilon \mathbf{c})\right]^{p}=\sum{ }^{\prime} I_{m, p}\left(G^{*}\right),
$$

where the summation in $\sum^{\prime}$ goes over the regular diagrams with $p$ levels. By computing the numbers of such diagrams, as in Breuer \& Major ([4], p. 434), we get

$$
\mathrm{E}\left[S_{g_{M}}(\varepsilon \mathbf{c})\right]^{p} \underset{\varepsilon \rightarrow 0}{\longrightarrow}(p) ! ! \sigma_{g_{M}, m}^{p}(\mathbf{c}, \mathbf{d}) .
$$

This achieves the proof of Lemma 4.1.

The following lemma studies the contribution of the irregular graphs.

Lemma 4.2 For fixed $j_{1}, \ldots, j_{p}$ we have

$$
L(\varepsilon):=\int_{[0,1]^{p}} \prod_{w \in G(V)}\left|\rho_{H}\left(\frac{s_{d_{1}(w)}-s_{d_{2}(w)}}{\varepsilon}, c_{j_{d_{1}(w)}}, c_{j_{d_{2}(w)}}\right)\right| d \mathbf{s}=o\left(\varepsilon^{p / 2}\right) .
$$

Proof of Lemma 4.2.

$$
L(\varepsilon)=\int_{[0,1]^{p}} \prod_{i=1}^{p}\left[\prod_{w \in G(V)} \prod_{d_{1}(w)=i}\left|\rho_{H}\left(\frac{s_{i}-s_{d_{2}(w)}}{\varepsilon}, c_{j_{i}}, c_{j_{d_{2}(w)}}\right)\right|\right] \mathrm{d} s_{i} .
$$


Let $K_{G}(i)$ be the number of edges such that $d_{1}(w)=i$,

$$
L(\varepsilon) \leq \int_{[0,1]^{p}} \prod_{i=1}^{p}\left[\frac{1}{K_{G}(i)} \sum_{w \in G(V)} \sum_{d_{1}(w)=i}\left|\rho_{H}\left(\frac{s_{i}-s_{d_{2}(w)}}{\varepsilon}, c_{j_{i}}, c_{j_{d_{2}(w)}}\right)\right|^{K_{G}(i)}\right] \mathrm{d} s_{i} .
$$

Let $A=\left\{\left(c_{j_{1}}, c_{j_{2}}\right), j_{1}=1, \ldots, m, j_{2}=1, \ldots, m\right\}$.

Using the same techniques as in the proposition of [4] p. 435,

$$
\begin{aligned}
L(\varepsilon) & \leq \prod_{i=1}^{p}\left[\sup _{(c, d) \in A} \sup _{v \in[0,1]} \int_{0}^{1}\left|\rho_{H}\left(\frac{s_{i}-v}{\varepsilon}, c, d\right)\right|^{K_{G}(i)} \mathrm{d} s_{i}\right] \\
& \leq \prod_{i=1}^{p}\left[\sup _{(c, d) \in A} \int_{-1}^{1}\left|\rho_{H}\left(\frac{u}{\varepsilon}, c, d\right)\right|^{K_{G}(i)} \mathrm{d} u\right] .
\end{aligned}
$$

Let $i$ be fixed in $\{1, \ldots, p\}$ and define $g(i)=\frac{K_{G}(i)}{l_{i}}$, with $c$ and $d$ held fixed in $A$. We have two cases to consider:

i) $K_{G}(i) \geq 1$

$$
\begin{aligned}
\int_{-1}^{1}\left|\rho_{H}\left(\frac{u}{\varepsilon}, c, d\right)\right|^{K_{G}(i)} \mathrm{d} u & =\varepsilon \int_{-\frac{1}{\varepsilon}}^{\frac{1}{\varepsilon}}\left|\rho_{H}(x, c, d)\right|^{K_{G}(i)} \mathrm{d} x \\
& \leq \varepsilon \int_{-\infty}^{\infty}\left|\rho_{H}(x, c, d)\right| \mathrm{d} x \\
& \leq \mathbf{C} \varepsilon=\mathbf{C} \varepsilon^{1-g(i)} \varepsilon^{g(i)} \leq \mathbf{C} \varepsilon^{g(i)}
\end{aligned}
$$

ii) $K_{G}(i)=0$

$$
\int_{-1}^{1}\left|\rho_{H}\left(\frac{u}{\varepsilon}, c, d\right)\right|^{K_{G}(i)} \mathrm{d} u=2=2 \varepsilon^{0}=2 \varepsilon^{g(i)}
$$

Thus

$$
L(\varepsilon) \leq \mathbf{C} \varepsilon^{\sum_{i=1}^{p} g(i)}
$$

As in [4] p. 436, either $\sum_{i=1}^{p} g(i)>p / 2$ (and in this case $L(\varepsilon)=o\left(\varepsilon^{p / 2}\right)$ ) or there exists $1 \leq i_{0} \leq p$ such that $0<K_{G}\left(i_{0}\right)<l_{i_{0}}$, i.e. $0<g\left(i_{0}\right)<1$, then

$$
L(\varepsilon) \leq \mathbf{C} \varepsilon^{\sum_{i=1}^{p} g(i)} \varepsilon^{\left(1-g\left(i_{0}\right)\right)},
$$

and since $\sum_{i=1}^{p} g(i)-p / 2 \geq 0$, Lemma 4.2 follows.

Hence, we have proved that

$$
S_{g_{M}}(\varepsilon \mathbf{c}) \underset{\varepsilon \rightarrow 0}{\Rightarrow} \mathcal{N}\left(0 ; \sigma_{g_{M}, m}^{2}(\mathbf{c}, \mathbf{d})\right) .
$$

Furthermore, $\sum_{n=M}^{\infty} \hat{g}_{n}^{2} n ! \underset{M \rightarrow \infty}{\longrightarrow} 0$, so we get

$$
\lim _{M \rightarrow \infty} \sup _{\varepsilon>0} \mathrm{E}\left[S_{g_{M}}(\varepsilon \mathbf{c})-S_{g}(\varepsilon \mathbf{c})\right]^{2}=0 .
$$

Now, since

$$
\mathcal{N}\left(0 ; \sigma_{g_{M}, m}^{2}(\mathbf{c}, \mathbf{d})\right) \underset{M \rightarrow \infty}{\Rightarrow} \mathcal{N}\left(0 ; \sigma_{g, m}^{2}(\mathbf{c}, \mathbf{d})\right),
$$

applying Lemma 1.1 of [6], Theorem 3.2 is proved. 


\subsection{Estimation of $H$}

\subsection{1 $\hat{H}_{\beta}$ estimator}

Proof of Corollary 3.2.

1) By using (6), (4) and (5) we get

$$
\begin{array}{r}
\beta\left(\hat{H}_{\beta}-2\right)=\sum_{i=1}^{l} z_{i}\left[(H-2) \beta \log \left(\varepsilon c_{i}\right)+\beta \log \left(\sigma_{2 H}\right)\right. \\
\left.+\log \left(\mathrm{E}\left[\left|N^{*}\right|^{\beta}\right]\right)+o_{\text {a.s. }, i}(1)\right]
\end{array}
$$

and property (7) gives

$$
\beta\left(\hat{H}_{\beta}-2\right)=(H-2) \beta+o_{a . s .}(1) .
$$

We proved that $\hat{H}_{\beta}$ is a strongly consistent estimator of $H$. Let us see now that $\hat{H}_{\beta}$ is an asymptotically unbiased estimator of $H$.

By (6)

$$
\beta\left(\mathrm{E}\left[\hat{H}_{\beta}\right]-2\right)=\sum_{i=1}^{l} z_{i} \mathrm{E}\left[\log \left(M_{\beta}\left(\varepsilon c_{i}\right)\right)\right]
$$

where $M_{\beta}(\varepsilon)$ is defined in (4). Since

$$
\int_{0}^{1}\left|Z_{\varepsilon}(u)\right|^{\beta} \mathrm{d} u=\frac{\varepsilon^{(2-H) \beta}}{\sigma_{2 H}^{\beta}} M_{\beta}(\varepsilon),
$$

by property (7), one has

$$
\begin{gathered}
\beta\left(\mathrm{E}\left[\hat{H}_{\beta}\right]-2\right)=\sum_{i=1}^{l} z_{i} \mathrm{E}\left[\log \left(\int_{0}^{1}\left|Z_{\varepsilon c_{i}}(u)\right|^{\beta} \mathrm{d} u\right)\right] \\
+(H-2) \beta
\end{gathered}
$$

Hence, it is enough to prove that

$$
\mathrm{E}\left[\log \left(\int_{0}^{1}\left|Z_{\varepsilon}(u)\right|^{\beta} \mathrm{d} u\right)\right] \underset{\varepsilon \rightarrow 0}{\longrightarrow} \log \left(\mathrm{E}\left[\left|N^{*}\right|\right]^{\beta}\right) .
$$

For this, let us notice that since $\log$ is a concave function and $\log (x) \leqslant x$ when $x \geqslant 0$, we have

$$
\int_{0}^{1} \log \left(\left|Z_{\varepsilon}(u)\right|^{\beta}\right) \mathrm{d} u \leqslant \log \left(\int_{0}^{1}\left|Z_{\varepsilon}(u)\right|^{\beta} \mathrm{d} u\right) \leqslant \int_{0}^{1}\left|Z_{\varepsilon}(u)\right|^{\beta} \mathrm{d} u .
$$

Thus, if we denote

$$
X_{\varepsilon}:=\int_{0}^{1}\left|Z_{\varepsilon}(u)\right|^{\beta} \mathrm{d} u+\beta\left|\int_{0}^{1} \log \left(\left|Z_{\varepsilon}(u)\right|\right) \mathrm{d} u\right|,
$$


we have shown that

$$
\left|\log \left(\int_{0}^{1}\left|Z_{\varepsilon}(u)\right|^{\beta} \mathrm{d} u\right)\right| \leqslant X_{\varepsilon} .
$$

Now, since $\left|N^{*}\right|^{\beta}$ and $\log \left(\left|N^{*}\right|\right)$ are in $L^{1}(\Omega)$, the same result is true for $X_{\varepsilon}$, say

$$
X_{\varepsilon} \in L^{1}(\Omega) .
$$

Furthermore by using Lemma 3.1 following Theorem 3.2, it is easy to see that

$$
X_{\varepsilon} \underset{\varepsilon \rightarrow 0}{\stackrel{\mathcal{L}^{1}}{\rightarrow}} \mathrm{E}\left[\left|N^{*}\right|^{\beta}\right]+\beta\left|\mathrm{E}\left[\log \left(\left|N^{*}\right|\right)\right]\right| .
$$

Finally, by using Theorem 3.1, we get:

$$
\log \left(\int_{0}^{1}\left|Z_{\varepsilon}(u)\right|^{\beta} \mathrm{d} u\right) \underset{\varepsilon \rightarrow 0}{\stackrel{a . s .}{\rightarrow}} \log \left(\mathrm{E}\left[\left|N^{*}\right|\right]^{\beta}\right) .
$$

Hence, (19), (20), (21) and (22) yield (18).

2) Formula (16) entails that

$$
\mathrm{E}\left[M_{\beta}(\varepsilon)\right]=\sigma_{2 H}^{\beta} \varepsilon^{(H-2) \beta} \mathrm{E}\left[\left|N^{*}\right|^{\beta}\right] .
$$

As in [7], let us define

$$
A_{\beta}(\varepsilon)=\frac{M_{\beta}(\varepsilon)-\mathrm{E}\left[M_{\beta}(\varepsilon)\right]}{\mathrm{E}\left[M_{\beta}(\varepsilon)\right]} .
$$

With this definition and using a Taylor expansion for the logarithm function one has

$$
\begin{aligned}
\log \left(M_{\beta}(\varepsilon)\right)= & \log \left(\mathrm{E}\left[M_{\beta}(\varepsilon)\right]\right)+\log \left(1+A_{\beta}(\varepsilon)\right) \\
= & (H-2) \beta \log (\varepsilon)+\beta \log \left(\sigma_{2 H}\right) \\
& +\log \left(\mathrm{E}\left[\left|N^{*}\right|^{\beta}\right]\right)+A_{\beta}(\varepsilon) \\
& +A_{\beta}^{2}(\varepsilon)\left[-\frac{1}{2}+\varepsilon_{1}\left(A_{\beta}(\varepsilon)\right)\right] .
\end{aligned}
$$

Let us see that

$$
A_{\beta}^{2}(\varepsilon)\left[-\frac{1}{2}+\varepsilon_{1}\left(A_{\beta}(\varepsilon)\right)\right]=o_{p}(\sqrt{\varepsilon}) .
$$

By the definition of $g_{\beta}$ (see (8)), one has

$$
A_{\beta}(\varepsilon)=\sqrt{\varepsilon} S_{g_{\beta}}(\varepsilon),
$$

and by Lemma 3.1 following Theorem 3.2

$$
\mathrm{E}\left[\frac{1}{\sqrt{\varepsilon}} A_{\beta}^{2}(\varepsilon)\right]=\sqrt{\varepsilon} \mathrm{E}\left[S_{g_{\beta}}^{2}(\varepsilon)\right]=O(\sqrt{\varepsilon}),
$$


so $\frac{1}{\sqrt{\varepsilon}} A_{\beta}^{2}(\varepsilon)=o_{p}(1)$ and then $(24)$ is proved.

By using (23) and (24) we obtain

$$
\begin{aligned}
\log \left(M_{\beta}(\varepsilon)\right)= & (H-2) \beta \log (\varepsilon)+\beta \log \left(\sigma_{2 H}\right) \\
& +\log \left(\mathrm{E}\left[\left|N^{*}\right|^{\beta}\right]\right)+\sqrt{\varepsilon} S_{g_{\beta}}(\varepsilon)+o_{p}(\sqrt{\varepsilon}) .
\end{aligned}
$$

Thus, by using (6) and property (7), we have

$$
\beta\left(\hat{H}_{\beta}-2\right)=(H-2) \beta+\sum_{i=1}^{l} z_{i} \sqrt{\varepsilon c_{i}} S_{g_{\beta}}\left(\varepsilon c_{i}\right)+o_{p}(\sqrt{\varepsilon}) .
$$

Thus

$$
\frac{\left(\hat{H}_{\beta}-H\right)}{\sqrt{\varepsilon}}=\frac{1}{\beta} \sum_{i=1}^{l} z_{i} \sqrt{c_{i}} S_{g_{\beta}}\left(\varepsilon c_{i}\right)+o_{p}(1) .
$$

Theorem 3.2 gives the required result.

Now, to conclude the proof of Corollary 3.2, we have to compute the coefficients in the Hermite expansion of function $g_{\beta}(x)=\frac{|x|^{\beta}}{\mathrm{E}\left[\left|N^{*}\right|^{\beta}\right]}-1$.

To simplify the notation, first let us compute the coefficients in the Hermite expansion of $h_{\beta}(x)=|x|^{\beta}-\mathrm{E}\left[\left|N^{*}\right|^{\beta}\right]$.

Since $h_{\beta}$ is even, we have: $h_{\beta}(x)=\sum_{n=1}^{\infty} \hat{h}_{2 n, \beta} H_{2 n}(x)$, with for $n \geqslant 1, \hat{h}_{2 n, \beta}=$ $\frac{2}{(2 n) !} \int_{0}^{+\infty} H_{2 n}(x) x^{\beta} \phi(x) \mathrm{d} x$.

By the definition of the Hermite polynomials and since for $a \geqslant 0$

$$
\Psi(a):=\int_{0}^{+\infty} x^{a} \phi(x) \mathrm{d} x=\pi^{-1 / 2} 2^{(a-2) / 2} \Gamma((a+1) / 2),
$$

we get

$$
\hat{h}_{2 n, \beta}=\pi^{-1 / 2} \sum_{p=0}^{n} \frac{(-1)^{(n-p)}}{2^{(n-p)}(n-p) !(2 p) !} 2^{(p+\beta / 2)} \Gamma(p+(\beta+1) / 2) .
$$

To obtain the first equality in (9), it suffices to see that $\hat{g}_{2 n, \beta}=\frac{\hat{h}_{2 n, \beta}}{2 \Psi(\beta)}$ and $(25)$ gives the required result. Moreover, Coeurjoly in [5], has shown that $\hat{g}_{2 n, \beta}=\frac{1}{(2 n) !} \prod_{i=0}^{n-1}(\beta-$ $2 i)$.

Proof of Remark 1 in Corollary 3.2. Let us note that $\hat{g}_{2, \beta}=\frac{\beta}{2}$. Then for $b, c>0$

$$
\rho_{g_{\beta}}(b, c)=\frac{\beta^{2}}{4} \rho_{g_{2}}(b, c)+\rho_{g_{\beta}^{\prime}}(b, c)
$$

where $g_{\beta}^{\prime}(x)=\sum_{n=2}^{\infty} \hat{g}_{2 n, \beta} H_{2 n}(x)$ which belongs to $L^{2}(\phi(x) \mathrm{d} x)$.

Then

$$
\begin{aligned}
\sigma_{g_{\beta}, l}^{2}(\mathbf{c}, \sqrt{\mathbf{c}}(\mathbf{z} / \beta)) & =\sigma_{g_{2}, l}^{2}(\mathbf{c}, \sqrt{\mathbf{c}}(\mathbf{z} / 2))+\sigma_{g_{\beta}^{\prime}, l}^{2}(\mathbf{c}, \sqrt{\mathbf{c}}(\mathbf{z} / \beta)) \\
& \geqslant \sigma_{g_{2}, l}^{2}(\mathbf{c}, \sqrt{\mathbf{c}}(\mathbf{z} / 2))
\end{aligned}
$$


since the last term in the above equality is positive by Lemma 3.1 following Theorem 3.2 .

\subsection{2 $\hat{H}_{\log }$ estimator}

Proof of Corollary 3.3.

1) Theorem 3.2 allows us to prove that

$$
\int_{0}^{1} \log \left(\left|Z_{\varepsilon}(u)\right|\right) \mathrm{d} u \underset{\varepsilon \rightarrow 0}{\stackrel{\mathcal{P}}{\longrightarrow}} \mathrm{E}\left[\log \left(\left|N^{*}\right|\right)\right] .
$$

Indeed, from the definition of the function $g_{\log }$ (see (12)), one has

$$
\int_{0}^{1} \log \left(\left|Z_{\varepsilon}(u)\right|\right) \mathrm{d} u=\mathrm{E}\left[\log \left(\left|N^{*}\right|\right)\right]+\sqrt{\varepsilon} S_{g_{\log }}(\varepsilon),
$$

and by Lemma 3.1 following Theorem 3.2,

$$
\mathrm{E}\left[\sqrt{\varepsilon} S_{g_{\log }}(\varepsilon)\right]^{2}=O(\varepsilon) .
$$

Thus (26) follows and from the definition of $M_{\log }(\varepsilon)$ (see (10)), one obtains

$$
M_{\log }(\varepsilon)=(H-2) \log (\varepsilon)+\log \left(\sigma_{2 H}\right)+\mathrm{E}\left[\log \left|N^{*}\right|\right]+o_{p}(1) .
$$

Now, by (11), (28) and using property (7), we get

$$
\hat{H}_{\log }-2=\sum_{i=1}^{l} z_{i} M_{\log }\left(\varepsilon c_{i}\right)=(H-2)+o_{p}(1) .
$$

We have proved that $\hat{H}_{\log }$ is a weakly consistent estimator of $H$. Let us show now that $\hat{H}_{\log }$ is unbiased.

Since

$$
\int_{0}^{1} \log \left(\left|Z_{\varepsilon}(u)\right|\right) \mathrm{d} u=(2-H) \log (\varepsilon)-\log \left(\sigma_{2 H}\right)+M_{\log }(\varepsilon)
$$

one has

$$
\mathrm{E}\left[M_{\log }(\varepsilon)\right]=\mathrm{E}\left[\log \left(\left|N^{*}\right|\right)\right]+(H-2) \log (\varepsilon)+\log \left(\sigma_{2 H}\right),
$$

and by (11) and property (7), we get

$$
\mathrm{E}\left[\hat{H}_{\log }\right]-2=\sum_{i=1}^{l} z_{i} \mathrm{E}\left[M_{\log }\left(\varepsilon c_{i}\right)\right]=H-2,
$$

and $\hat{H}_{\log }$ is an unbiased estimator of $H$. 
2) Now, (29), (30), (27) and (31) entail that

$$
\begin{aligned}
\hat{H}_{\log }-2 & =\sum_{i=1}^{l} z_{i}\left(M_{\log }\left(\varepsilon c_{i}\right)-\mathrm{E}\left[M_{\log }\left(\varepsilon c_{i}\right)\right]\right)+\sum_{i=1}^{l} z_{i} \mathrm{E}\left[M_{\log }\left(\varepsilon c_{i}\right)\right] \\
& =\sum_{i=1}^{l} z_{i} \sqrt{\varepsilon c_{i}} S_{g_{\log }}\left(\varepsilon c_{i}\right)+(H-2) .
\end{aligned}
$$

Thus

$$
\frac{\left(\hat{H}_{\log }-H\right)}{\sqrt{\varepsilon}}=\sum_{i=1}^{l} z_{i} \sqrt{c_{i}} S_{g_{\log }}\left(\varepsilon c_{i}\right) .
$$

Theorem 3.2 gives the required result.

Now, to conclude the proof of Corollary 3.3, we have to compute the coefficients in the Hermite expansion of function $g_{\log }(x)=\log (|x|)-\mathrm{E}\left[\log \left(\left|N^{*}\right|\right)\right]$. As before, $g_{\log }(x)=\sum_{n=1}^{\infty} \hat{g}_{2 n, \log } H_{2 n}(x)$ with

$$
\hat{g}_{2 n, \log }=\frac{2}{(2 n) !} \int_{0}^{+\infty} H_{2 n}(x) \log (x) \phi(x) d x=\frac{2}{(2 n) !} \int_{0}^{+\infty} H_{2 n-1}(x) \frac{1}{x} \phi(x) d x .
$$

For the last integral we used that $H_{m}(x) \phi(x)=(-1)^{m} \frac{d^{m}}{d x^{m}}(\phi(x))$, and integration by parts. Now the definition of the Hermite polynomials and (25) yield

$$
\hat{g}_{2 n, \log }=\frac{\pi^{-1 / 2}}{(2 n)} \sum_{p=0}^{n-1} \frac{(-1)^{(n-1-p)}}{2^{(n-1-2 p)}(n-1-p) !(2 p+1) !} \Gamma(p+1 / 2) .
$$

Consequently, by formula for $\Gamma(p+1 / 2)$, one has

$$
\begin{aligned}
\hat{g}_{2 n, \log } & =\frac{1}{2^{n} n !} \sum_{p=0}^{n-1}(-1)^{(n-1-p)} C_{n-1}^{p}\left(\frac{1}{2 p+1}\right) \\
& =\frac{(-1)^{n-1}}{2^{n} n !} \int_{0}^{1}\left(1-x^{2}\right)^{n-1} d x=\frac{(-1)^{(n-1)}}{2 n(2 n) ! !}
\end{aligned}
$$

which gives the required result.

\subsection{3 $\hat{H}_{\beta(\varepsilon)}$ estimator}

Proof of Corollary 3.4. Let

$$
\tilde{S}(\varepsilon)=\frac{1}{\beta(\varepsilon)} S_{g_{\beta(\varepsilon)}}(\varepsilon)
$$

where

$$
g_{\beta(\varepsilon)}(x)=\frac{|x|^{\beta(\varepsilon)}}{\mathrm{E}\left[\left|N^{*}\right| \beta(\varepsilon)\right]}-1=\sum_{n=1}^{\infty} \hat{g}_{2 n, \beta(\varepsilon)} H_{2 n}(x) .
$$

As in [5], we have the following lemma 


\section{Lemma 4.3}

$$
\tilde{S}(\varepsilon)-S_{g_{\log }}(\varepsilon) \underset{\varepsilon \rightarrow 0}{\stackrel{\mathcal{L}^{2}}{\rightarrow}} 0 .
$$

Proof of Lemma 4.3. Lemma 3.1 following Theorem 3.2 gives the asymptotic behavior of $\mathrm{E}\left[S_{g_{\log }}(\varepsilon)\right]^{2}$, set

$$
\mathrm{E}\left[S_{g_{\log }}(\varepsilon)\right]^{2} \underset{\varepsilon \rightarrow 0}{\longrightarrow} \sigma_{g_{\log }}^{2}
$$

Now, (9) in Corollary 3.2 gives the expression of the Hermite's coefficients, $\hat{g}_{2 n, \beta(\varepsilon)}$, of $g_{\beta(\varepsilon)}$, let

$$
\hat{g}_{2 n, \beta(\varepsilon)}=\frac{1}{(2 n) !} \prod_{i=0}^{n-1}(\beta(\varepsilon)-2 i)
$$

and as in the proof of Lemma 3.1 following Theorem 3.2, Mehler's formula (3) allows us to compute $\mathrm{E}[\tilde{S}(\varepsilon)]^{2}$, say

$$
\mathrm{E}[\tilde{S}(\varepsilon)]^{2}=\sum_{n=1}^{\infty}\left(\frac{\hat{g}_{2 n, \beta(\varepsilon)}}{\beta(\varepsilon)}\right)^{2}(2 n) ! \int_{-1 / \varepsilon}^{1 / \varepsilon}(1-\varepsilon|x|) \rho_{H}^{2 n}(x) \mathrm{d} x .
$$

The expression of the Hermite coefficients, $\hat{g}_{2 n, \log }$, of $g_{\log }$ is given in (13) in Corollary 3.3 and we observe that

$$
\frac{\hat{g}_{2 n, \beta(\varepsilon)}}{\beta(\varepsilon)} \underset{\varepsilon \rightarrow 0}{\longrightarrow} \frac{1}{(2 n) !} \prod_{i=1}^{n-1}(-2 i)=\hat{g}_{2 n, \log } .
$$

Furthermore for small enough $\varepsilon$

$$
\left|\frac{\hat{g}_{2 n, \beta(\varepsilon)}}{\beta(\varepsilon)}\right|=\frac{1}{(2 n) !} \prod_{i=1}^{n-1}(2 i-\beta(\varepsilon)) \leqslant \frac{1}{(2 n) !} \prod_{i=1}^{n-1}(2 i)=\left|\hat{g}_{2 n, \log }\right| .
$$

Since $\left\|g_{\log }\right\|_{2, \phi}^{2}<+\infty$, we get

$$
\mathrm{E}[\tilde{S}(\varepsilon)]^{2} \underset{\varepsilon \rightarrow 0}{\longrightarrow} \sigma_{g_{\log }}^{2}
$$

To achieve the proof of Lemma 4.3, we compute $\mathrm{E}\left[\tilde{S}(\varepsilon) S_{g_{\log }}(\varepsilon)\right]$ by Mehler's formula and we proceed as for $\mathrm{E}[\tilde{S}(\varepsilon)]^{2}$ to obtain

$$
\mathrm{E}\left[\tilde{S}(\varepsilon) S_{g_{\log }}(\varepsilon)\right] \underset{\varepsilon \rightarrow 0}{\longrightarrow} \sigma_{g_{\log }}^{2},
$$

(32), (33) and (34) give the required result.

Let us show now that from the definition of $M_{\beta(\varepsilon)}(\varepsilon)$ given in (14), one has

$$
\begin{aligned}
\frac{\log \left(M_{\beta(\varepsilon)}(\varepsilon)\right)}{\beta(\varepsilon)}= & (H-2) \log (\varepsilon)+\log \left(\sigma_{2 H}\right) \\
& +\mathrm{E}\left[\log \left(\left|N^{*}\right|\right)\right]+\sqrt{\varepsilon} \tilde{S}(\varepsilon)+o_{p}(\sqrt{\varepsilon})
\end{aligned}
$$

Indeed, as in the proof of Corollary 3.2, we get

$$
\frac{\log \left(M_{\beta(\varepsilon)}(\varepsilon)\right)}{\beta(\varepsilon)}=(H-2) \log (\varepsilon)+\log \left(\sigma_{2 H}\right)
$$




$$
+\frac{\log \left(\mathrm{E}\left[\left|N^{*}\right|^{\beta(\varepsilon)}\right]\right)}{\beta(\varepsilon)}+\sqrt{\varepsilon} \tilde{S}(\varepsilon)+o_{p}(\sqrt{\varepsilon}) .
$$

Since

$$
\frac{\log \left(\mathrm{E}\left[\left|N^{*}\right|^{\beta(\varepsilon)}\right]\right)}{\beta(\varepsilon)}-\mathrm{E}\left[\log \left(\left|N^{*}\right|\right)\right]=O(\beta(\varepsilon)),
$$

and $\beta(\varepsilon)=o(\sqrt{\varepsilon}),(35)$ holds.

Thus we follow the same lines as in the proof of Corollary 3.2 to obtain

$$
\frac{\left(\hat{H}_{\beta(\varepsilon)}-H\right)}{\sqrt{\varepsilon}}=\sum_{i=1}^{l} z_{i} \sqrt{c_{i}} \tilde{S}\left(\varepsilon c_{i}\right)+o_{p}(1),
$$

where $\hat{H}_{\beta(\varepsilon)}$ is defined in (14). Lemma 4.3 and Corollary 3.3 allow us to conclude the asymptotic normality of $\hat{H}_{\beta(\varepsilon)}$.

Let us show now that $\hat{H}_{\beta(\varepsilon)}$ is an asymptotically unbiased estimator of $H$. As in the proof of Corollary 3.2 (see (17) and (18)), it is enough to prove that

$$
\frac{1}{\beta(\varepsilon)} \mathrm{E}\left[\log \left(\int_{0}^{1}\left|Z_{\varepsilon}(u)\right|^{\beta(\varepsilon)} \mathrm{d} u\right)\right] \underset{\varepsilon \rightarrow 0}{\longrightarrow} \mathrm{E}\left[\log \left(\left|N^{*}\right|\right)\right] .
$$

To show this above convergence, we use the fact that log is a concave function and then

$$
\begin{aligned}
\beta(\varepsilon) \mathrm{E}\left[\log \left(\left|N^{*}\right|\right)\right] & =\mathrm{E}\left[\int_{0}^{1} \log \left(\left|Z_{\varepsilon}(u)\right|^{\beta(\varepsilon)}\right) \mathrm{d} u\right] \\
& \leqslant \mathrm{E}\left[\log \left(\int_{0}^{1}\left|Z_{\varepsilon}(u)\right|^{\beta(\varepsilon)} \mathrm{d} u\right)\right] \\
& \leqslant \log \left(\mathrm{E}\left[\int_{0}^{1}\left|Z_{\varepsilon}(u)\right|^{\beta(\varepsilon)} \mathrm{d} u\right]\right) \\
& =\log \left(\mathrm{E}\left[\left|N^{*}\right|^{\beta(\varepsilon)}\right]\right) .
\end{aligned}
$$

If we divide both sides, in the above inequality, by $\beta(\varepsilon)$ and using (36), we get (37).

Final remark: We have considered the estimation of the Hurst parameter for fractional Brownian motion. The same procedure can be implemented, with little changes, when the observed process is a Gaussian process with stationary increments. In this case the incremental variance must be: $E\left(X_{t+h}-X_{t}\right)^{2}=h^{H} L(h)$ for $L(\cdot)$ a slowly varying function in zero. Here the estimation refers to the $H$ parameter. 


\section{References}

[1] Jean-Marc Azaïs and Mario Wschebor. Almost sure oscillation of certain random processes. Bernoulli, 2(3): 257-270, 1996.

[2] A. Benassi, S. Cohen, J. Istas and S. Jaffard. Identification of Filtered White Noises. Stoch. Proc. Appl., 75(1): 31-50, 1998.

[3] Corinne Berzin and José R. León. Convergence in fractional models and applications. Electronic Journal of Probability, to appear, 2005.

[4] Peter Breuer and Peter Major. Central limit theorems for non-linear functionals of Gaussian fields. J. Multivariate Anal., 13: 425-441, 1983.

[5] Jean-François Coeurjolly. Estimating the parameters of a fractional Brownian motion by discrete variations of its sample paths. Statistical Inference for Stochastic Processes, 4: 199-277, 2001.

[6] E.B. Dynkin. Self-intersection gauge for random walks and for Brownian motion. Ann. Probab., 16(1): 1-57, 1988.

[7] Andrey Feuerverger, Peter Hall and Andrew T. A. Wood. Estimation of fractal index and fractal dimension of a Gaussian process by counting the number of level crossings. Journal of time series analysis, 15(6): 587-606, 1994.

[8] P. Flandrin. Wavelet analysis and synthesis of fractional Brownian motion. IEEE Transactions on Information Theory, 38: 910-917, 1992.

[9] R. Fox and M.S. Taqqu. Large-sample properties of parameter estimates for strongly dependent Gaussian stationary time series. Ann. of Statistics, 14(6): 517-532, 1986.

[10] Hwai-Chung Ho and Tze-Chien Sun. Limiting distributions of nonlinear vector functions of stationary Gaussian processes. Ann. Probab., 18(3): 1159-1173, 1990.

[11] J. Istas and G. Lang. Quadratic variations and estimation of the local Hölder index of a gaussian process. Ann. Inst. Poincaré, 33(4): 407-436, 1997.

[12] C. Ludeña. Parametric estimation for Gaussian long-range dependent processes based on the log-periodogram. Bernoulli, 6(4): 709-728, 2000.

[13] B.B. Mandelbrot and J.W. Van Ness. Fractional Brownian motions, fractional noises and applications. SIAM Rev., 10: 422-437, 1968.

[14] E. Moulines and P. Soulier. Broad band log-periodogram regression of time series with long range dependence. Ann. of Statistics, 27: 1415-1439, 1999.

[15] C. Velazco and P.M. Robinson. Whittle pseudo-maximun likelihood estimation of nonstationary time series. JASA, 95: 1229-1243, 2000. 